\author{
Shigeru Ohtsubo · Aritoshi Iida $\cdot$ Kosaku Nitta \\ Toshihiro Tanaka - Ryo Yamada - Yozo Ohnishi \\ Shiro Maeda $\cdot$ Tatsuhiko Tsunoda $\cdot$ Takashi Takei \\ Wataru Obara · Fumihiro Akiyama · Kyoko Ito \\ Kazuho Honda · Keiko Uchida - Ken Tsuchiya \\ Wako Yumura · Takashi Ujiie · Yutaka Nagane \\ Satoru Miyano · Yasushi Suzuki · Ichiei Narita \\ Fumitake Gejyo - Tomoaki Fujioka - Hiroshi Nihei \\ Yusuke Nakamura

\section{Association of a single-nucleotide polymorphism in the immunoglobulin $\mu$-binding protein 2 gene with immunoglobulin A nephropathy}

Received: 22 September 2004 / Accepted: 21 October 2004/Published online: 14 December 2004

(C) The Japan Society of Human Genetics and Springer-Verlag 2004

\begin{abstract}
Immunoglobulin A (IgA) nephropathy is the most common form of primary glomerulonephritis worldwide. The pathogenesis of $\operatorname{IgA}$ nephropathy is unknown, but it is certain that some genetic factors are involved in susceptibility to the disease. Employing a large-scale, case-control association study using genebased single-nucleotide polymorphism (SNP) markers, we previously reported four candidate genes. We report here an additional significant association between $\operatorname{IgA}$ nephropathy and an SNP located in the gene encoding immunoglobulin $\mu$-binding protein 2 (IGHMBP2) at
\end{abstract}

S. Ohtsubo $\cdot$ S. Miyano $\cdot$ Y. Nakamura $(\square)$

Human Genome Center, The Institute of Medical Science,

University of Tokyo, 4-6-1 Shirokanedai, Minato-ku,

Tokyo 108-8639, Japan

E-mail: yusuke@ims.u-tokyo.ac.jp

Tel.: + 81-3-54495372

Fax: $+81-3-34495433$

S. Ohtsubo $\cdot$ K. Nitta $\cdot$ T. Takei $\cdot$ K. Ito $\cdot$ K. Honda $\cdot$ K. Uchida

K. Tsuchiya $\cdot$ W. Yumura $\cdot H$. Nihei

Department of Medicine, Kidney Center,

Tokyo Women's Medical University, Tokyo, Japan

A. Iida

Laboratory for Genotyping, SNP Research Center,

The Institute of Physical and Chemical Research (RIKEN),

Tokyo, Japan

T. Tanaka $\cdot$ Y. Ohnishi

Laboratory for Cardiovascular Diseases, SNP Research Center,

The Institute of Physical and Chemical Research (RIKEN),

Tokyo, Japan

R. Yamada

Laboratory for Rheumatic Diseases, SNP Research Center,

The Institute of Physical and Chemical Research (RIKEN),

Tokyo, Japan chromosome 11q13.2-q13.4. The association $\left(\chi^{2}=17.1\right.$, $p=0.00003$; odds ratio of 1.85 with $95 \%$ confidence interval of $1.39-2.50$ in a dominant association model) was found using DNA from 465 affected individuals and 634 controls. The SNP (G34448A) caused an amino acid substitution from glutamine to lysine (E928K). As the gene product is involved in immunoglobulin-class switching and patients with the A allele revealed higher serum levels of $\operatorname{IgA}(p=0.048)$, the amino acid change might influence a class switch to increase serum IgA levels, resulting in a higher risk of $\operatorname{IgA}$ nephropathy.

\section{S. Maeda}

Laboratory for Diabetic Nephropathy, SNP Research Center,

The Institute of Physical and Chemical Research (RIKEN),

Tokyo, Japan

T. Tsunoda

Laboratory for Medical Informatics, SNP Research Center,

The Institute of Physical and Chemical Research (RIKEN),

Tokyo, Japan

W. Obara $\cdot$ Y. Suzuki $\cdot$ T. Fujioka

Department of Urology, Iwate Medical University,

Iwate, Japan

F. Akiyama · I. Narita · F. Gejyo

Division of Clinical Nephrology and Rheumatology,

Niigata University Graduate School of Medical

and Dental Sciences, Niigata, Japan

T. Ujiie

Department of Urology,

Iwate Prefectural Ofunato Hospital,

Iwate, Japan

Y. Nagane

Department of Urology,

Sanai Hospital, Iwate, Japan 
Keywords Single-nucleotide polymorphism $\cdot \operatorname{IgA}$ nephropathy $\cdot$ Immunoglobulin $\mu$-binding protein 2

\section{Introduction}

Immunoglobulin A (IgA) nephropathy, originally described by Berger and Hinglasis (1968), is now recognized as the most common form of primary glomerulonephritis worldwide. This disease is defined as a process of proliferative changes in glomerular mesangial cells and increases in mesangial matrices associated with deposition of IgA. At first, IgA nephropathy was considered to be a mild clinical manifestation with a relatively good prognosis. However, since recent reports have indicated that $20-30 \%$ of patients develop end-stage renal disease within 10 30 years after the onset of the disease (Koyama et al. 1997), its prognosis is now regarded as more serious than previously thought.

The pathogenesis of $\operatorname{IgA}$ nephropathy is unknown, but accumulating evidence suggests that genetic factors are involved in disease susceptibility (Donadio and Grande 2002). For example, the prevalence of IgA nephropathy seems to reflect demographic and ethnic characteristics of the populations studied; furthermore, several cases of familial IgA nephropathy have been reported (Scolari 2003).

Recent approaches to identify disease-susceptibility genes have focused on genome-wide analysis of associations using single-nucleotide polymorphisms (SNPs). SNPs are likely to be useful as markers for identification of genes involved in complex diseases because they are the most common type of genetic variation in human DNA (Kruglyak 1999). We have been screening gene-based SNPs on a genome-wide scale to detect possible associations with susceptibility to $\operatorname{IgA}$ nephropathy and have already reported that specific SNP alleles in the L- and E-selectin (SELL and SELE) genes on chromosome 1q24-25 (Takei et al. 2002), the $H L A-D R A$ locus at $6 \mathrm{p} 21.3$ (Akiyama et al. 2002), and the polymeric immunoglobulin receptor $(P I G R)$ gene at 1q31-q41 (Obara et al. 2003) were candidate susceptibility factors for IgA nephropathy in the Japanese population.

Through an extension of those genome-wide analyses, we have now identified an additional association with $\operatorname{IgA}$ nephropathy in the Japanese population involving SNPs in the immunoglobulin $\mu$-binding protein $2(I G H M B P 2)$ gene.

\section{Materials and methods}

DNA samples

This study enrolled 465 Japanese individuals with IgA nephropathy who were referred to several hospitals in
Japan (Department of Medicine, Kidney Center, Tokyo Women's Medical University; Department of Urology, Iwate Medical University; Division of Clinical Nephrology and Rheumatology, Niigata University Graduate School of Medical and Dental Sciences; Iwate Prefectural Ofunato Hospital; Sanai Hospital; Osaka City General Hospital; Toride Kyodo General Hospital; and Osaka Rosai Hospital). IgA nephropathy was diagnosed on the basis of clinical manifestations as well as renal-biopsy findings. Patients with Henoch-Schönlein purpura and secondary $\operatorname{IgA}$ nephropathy, such as hepatic glomerulosclerosis, were excluded from the analysis. Peripheral blood samples were obtained for DNA extraction. As controls, we analyzed DNA from 634 volunteers from the general Japanese population. Written informed consent was obtained from all participants, and DNA was prepared from each blood sample according to standard protocols. We are given approval for the study using human materials from our committee dealing with ethics for medical research.

\section{Multiplexed polymerase chain reactions}

We amplified multiple genomic fragments using $20 \mathrm{ng}$ of genomic DNA for each polymerase chain reaction (PCR), as described previously (Ohnishi et al. 2001). Sequences of the primers used in this study are available from the JSNP database (http://snp.ims.u-tokyo.ac.jp/). Each PCR was performed in a $20-\mu$ solution containing 50 pmol of each primer, $10 \mathrm{U}$ Ex-Taq DNA polymerase (TaKaRa), and $0.55 \mu \mathrm{g}$ TaqStart (Clontech Laboratories) in a GeneAmp PCR system 9700 (Applied Biosystems). Initial denaturation was performed at $94^{\circ} \mathrm{C}$ for $2 \mathrm{~min}$, followed by 37 cycles of amplification at $94^{\circ} \mathrm{C}$ for $15 \mathrm{~s}$ and annealing at $60^{\circ} \mathrm{C}$ for $45 \mathrm{~s}$, with final extension for $2 \mathrm{~min}$ at $72^{\circ} \mathrm{C}$.

\section{Genotyping}

For a new genome-wide association study, we selected 88,148 SNPs described in our JSNP database (Haga et al. 2002). All genotyping was done by the Invader assay, which combines a structure-specific cleavage enzyme with a universal fluorescent resonance energy transfer (FRET) system (Mein et al. 2000). FRET probes were labeled with either FAM or VIC corresponding to each allele. Signal intensity was indicated as the ratio of FAM or VIC to ROX, an internal reference. Each $5-\mu 1$ reaction contained $0.25 \mu 1$ of signal buffer, $0.25 \mu \mathrm{l}$ of each FRET probe, $0.25 \mu \mathrm{l}$ of the structure-specific cleavage enzyme, $0.25 \mu \mathrm{l}$ of allelespecific probe mix, and $2 \mu \mathrm{l}$ of the PCR product diluted 1:10. Samples were incubated in a GeneAmp PCR system $9700\left(95^{\circ} \mathrm{C}\right.$ for $5 \mathrm{~min}$ and then at $63^{\circ} \mathrm{C}$ for $15 \mathrm{~min}$ ) and were analyzed on an ABI Prism 7700 sequence detector. 
Statistical analysis

The genotype distribution and allele frequencies of each selected SNP were compared between cases and controls by means of chi ${ }^{2}$ tests. Significance was judged according to the guidelines of Lander and Kruglyak (1995). Fisher's exact test was used when criteria for the chi-squared test could not be applied. Odds ratios (OR) and 95\% confidence intervals $(95 \%$ CI $)$ were calculated by Woolf's method. Hardy-Weinberg equilibrium was assessed by chi-squared statistics (Nielsen et al. 1998). Linkage disequilibrium (LD) coefficients were calculated and expressed as $D^{\prime}=D / D_{\max }$ (Devlin and Risch 1995). Clinical data were recorded as means \pm SD. Statistically significant differences were assessed with Student's $t$ tests using Stat View SE.

\section{Results}

We performed this genome-wide association study in a stepwise manner. First, we genotyped 94 Japanese patients with IgA nephropathy for 88,148 gene-based SNPs selected from our database (Haga et al. 2002) by means of the high-throughput multiplex PCR-Invader assay method (Ohnishi et al. 2001). A comparison of the allelic frequencies among these 94 patients with frequencies calculated for 634 members of the general Japanese population identified 2,109 SNPs that revealed $p$ values of 0.01 or smaller. We then genotyped these 2,109 SNPs using DNAs of 94 additional patients (Fig. 1) and found that 581 of them still revealed $P$ values of 0.01 or smaller.

When these 581 polymorphisms were analyzed for all 465 cases, 13 of the SNPs, including those reported previously in the HLA-DRA gene, revealed $p$ values of 0.0001 or smaller. One of the SNPs showing relatively more significant associations was located in exon 14 of the immunoglobulin $\mu$-binding protein 2 (IGHMBP2) locus on chromosome 11q13.2-q13.4. This SNP, G34448A, was associated with IgA nephropathy with $\chi^{2}$ $=17.1, P=0.00003$ (odds ratio $=1.85,95 \% \mathrm{CI}=1.39$ 2.50 ) by a dominant association model (Table 1). The $\mathrm{G}$ to A substitution at nucleotide 34448 changed glutamine to lysine (E928K) (Fig. 2).

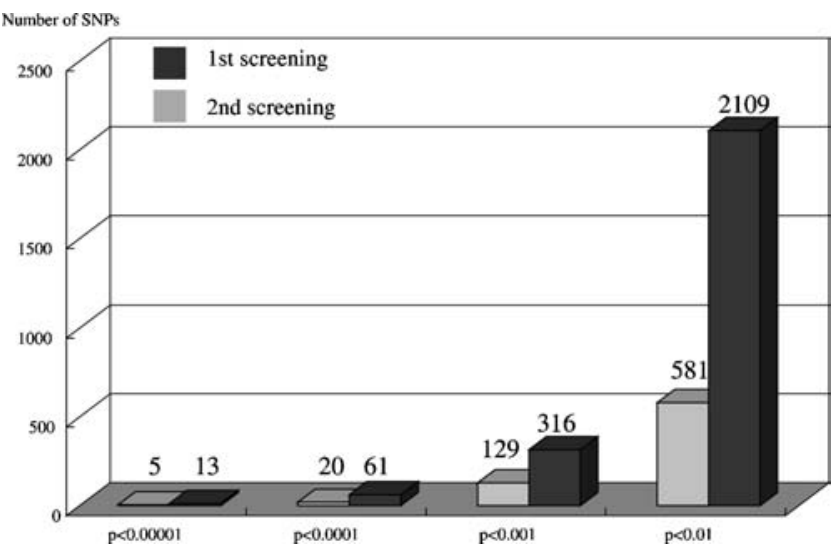

Fig. 1 Results of the first and second screening experiments. First screening (94 cases), $P<0.01: 2,109 / 88,148$ SNPs; second screening (188 cases), $P<0.01$ : 581/2,109 SNPs. By the increase of the number of the cases examined, most of the SNPs lost their association

We subsequently carried out LD mapping of 21 SNPs with minor allele frequencies of $>20 \%$ that were located in the IGHMBP2 region; this genomic fragment included six other genes (MTL5, CPT1A, MRPL21, $M R G P R D, M R G P R F$, and TPCN2). Mapping procedures separated these 21 SNPs into three LD blocks; the candidate SNP was located in the LD block containing two genes, MRPL21 and IGHMBP2. When we genotyped patients with IgA nephropathy for the eight SNPs present in that LD block, G34448A in exon 14 of $I G$ $H M B P 2$ showed the most significant association with the disease (Fig. 3).

Since individuals having the AA or GA genotype (homozygotes for the minor allele, or heterozygotes) of G34448A were likely to have a higher risk of IgA nephropathy $(P=0.00003)$ in a dominant manner, we compared available clinical data at the time of biopsy for patients with A alleles (AA and GA genotype) with those with the GG genotype (Table 2). We found no significant differences with respect to gender, age, levels of serum creatinine or 24-h urinary excretion of protein, but observed higher serum IgA levels in patients with AA and GA genotypes than in subjects with the GG genotype $(P=0.048)$.
Table 1 Genotype frequencies and association tests of the SNP in the IGHMBP2 gene (465 cases of IgA versus 634 control)

\begin{tabular}{|c|c|c|c|}
\hline & IgA number $(\%)$ & Control number $(\%)$ & \\
\hline Major allele & $798(85.8 \%)$ & $1162(91.6 \%)$ & \\
\hline Minor allele & $132(14.2 \%)$ & $106(8.3 \%)$ & \\
\hline Total & $930(100 \%)$ & $1268(100 \%)$ & \\
\hline Major homozygous & $342(73.5 \%)$ & $531(83.8 \%)$ & \\
\hline Heterozygous & $114(24.5 \%)$ & $100(15.8 \%)$ & \\
\hline Minor homozygous & $9(1.9 \%)$ & $3(0.47 \%)$ & \\
\hline \multirow[t]{2}{*}{ Total } & $465(100 \%)$ & $634(100 \%)$ & \\
\hline & $\chi^{2}$ & $P$ & Odds ratio $(95 \% \mathrm{CI})$ \\
\hline Allele frequency (minor versus major) & 18.9 & 0.00001 & $1.81(1.38-2.38)$ \\
\hline Other versus major homozygous & 17.1 & 0.00003 & $1.85(1.39-2.50)$ \\
\hline Minor homozygous versus other & 5.3 & $0.0353^{\mathrm{a}}$ & $3.90(1.03-14.77)$ \\
\hline
\end{tabular}


Fig. 2 Genomic structure of chromosome 11q13.2-q13.4 around the IGHMBP2 gene. $I G H M B P 2$ gene contains 36687 bp and 15 exons. The $34448 \mathrm{G} / \mathrm{A}$ SNP lies in exon 14 of IGHMBP2 (upward arrow). The $\mathrm{G}$ to A substitution at nucleotide 34448 changed an amino acid from glutamine to lysine (E928K); exons are indicated by black rectangles. The SNP number is the same as Fig. 3

Fig. 3 Pairwise linkage disequilibrium between SNPs in the $I G H M B P 2$ region, as measured by $D^{\prime}$ in the case population and case-control association plots $\left[-\log _{10}(P\right.$ value)]. The candidate SNP was located in the LD block containing two genes, MRPL21 and IGHMBP2. G34448A in exon 14 of $I G H M B P 2$ showed the most significant association with the disease in this block. The SNP number is the same as Fig. 2
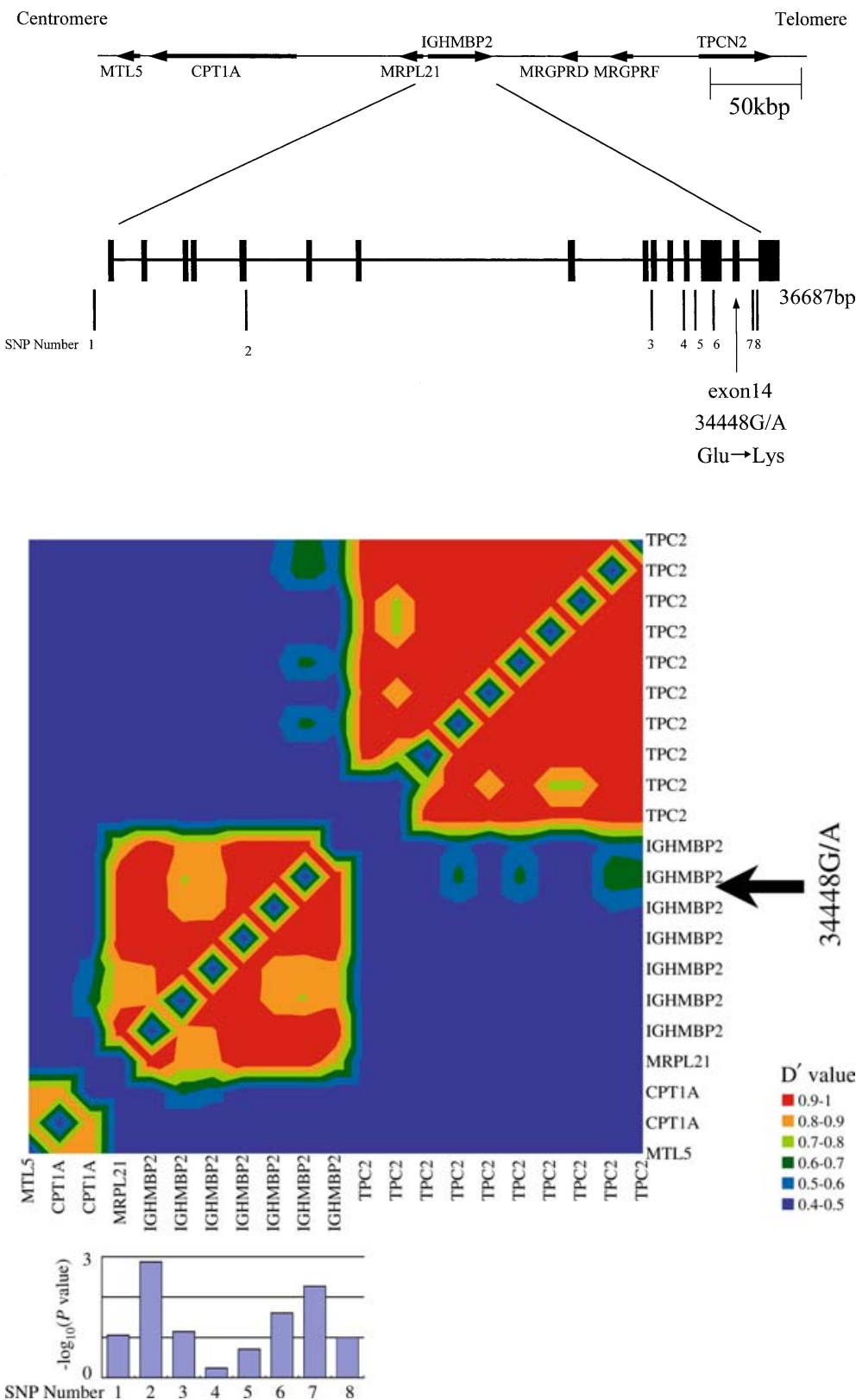

Table 2 Clinical data at the time of biopsy. Mean $\pm \mathrm{SD}, N S$ not significant

\begin{tabular}{lccl}
\hline & GG & GA + AA & $P$ value \\
\hline Gender (M/F) & $140 / 122$ & $60 / 38$ & NS \\
Age (years) & $35.9 \pm 14.2$ & $38.9 \pm 14.9$ & NS \\
Serum IgA (mg/dl) & $319.4 \pm 117.6$ & $352.4 \pm 162.9$ & 0.048 \\
Serum creatinine (mg/dl) & $1.06 \pm 0.66$ & $1.08 \pm 0.67$ & NS \\
Proteinuria (g/day) & $1.16 \pm 1.33$ & $1.04 \pm 1.33$ & NS \\
\hline
\end{tabular}

\section{Discussion}

Our genome-wide SNP system offers at least two advantages. First, our SNP database is an extensive catalog of gene-based SNPs located within exons, in- trons, and promoter regions (Haga et al. 2002). Second, using a combination of multiplex PCR and the Invader assay, our method allows high-throughput, low-cost screening that requires as little as $0.1 \mathrm{ng}$ of genomic DNA per single SNP. Through a genomewide SNP analysis, we found a significant association between IgA nephropathy and an SNP in the $I G$ $H M B P 2$ gene. This polymorphism, G34448A, substitutes lysine for glutamine in the gene product; the allele encoding lysine was more frequently observed in individuals with $\operatorname{IgA}$ nephropathy than in the general Japanese population. IGHMBP2 is ubiquitously expressed and consists of 15 exons encoding a protein of 993 amino acids that possesses a putative DNA helicase region, an $\mathrm{R} 3 \mathrm{H}$ motif, and a zinc-finger AN1-like domain. 
Although the physiological function of IGHMBP2 is not known in detail, the protein is suspected to be involved in immunoglobulin-class switching, pre-mRNA processing, and regulation of transcription through DNA-binding or interaction with TATA-binding proteins (Fukita et al. 1993; Molnar et al. 1997; Zhang et al. 1999; Miao et al. 2000; Liepinsh 2003). Fukita et al. (1993) first showed that IGHMBP2 binds to singlestranded DNA with 5'-phosphorylated guanine-rich sequences related to the immunoglobulin $\mu$-chain switch (Sm) region. Immunoglobulin switch (S) regions are essential targets of $\mathrm{S}-\mathrm{S}$ recombination, which changes the expressed class (isotype) of the immunoglobulin heavy chain $(\mathrm{H})$ constant region (C) from $\mu$ to $\gamma, \varepsilon$, or $\alpha$ while maintaining the antigen specificity determined by DNA sequences in the variable region. $\mathrm{S}$ regions are located $5^{\prime}$ to each $\mathrm{C}_{\mathrm{H}}$ gene, except for $\mathrm{C}_{\delta}$.

Increases in serum IgA levels have been observed in $50-70 \%$ of patients with IgA nephropathy, along with selective deposition of IgA1 in the kidney (Conley et al. 1980; Trascasa et al. 1980). We found that patients with the A allele of G34448A had higher serum IgA levels $(P=0.048)$ and suspected that the E928K amino acid substitution might affect the class-switch event thereby increasing serum IgA levels, elevating the risk of IgA deposition, and causing the disease. However, the biological role of the zinc-finger AN1-like domain, where the E928K substitution lies, is still unknown. To clarify the relationship between E928K amino acid substitution and mesangial IgA deposition, examination of the frequency of this SNP in another nephritis with the same deposition, such as Henoch-Schönlein purpura, might be effective, but we do not have any available sample.

Gharavi et al. (2000) reported a genome-wide analysis of familial cases, but no familial history of the disease was observed in most Japanese patients with IgA nephropathy. This disease is a complex disorder, the etiology of which involves immunological, environmental, and genetic factors (Hsu et al. 2000). The fact that mesangial IgA deposition has been observed in $16 \%$ of allografts in non-blood-related Japanese kidney donors (Suzuki et al. 2003) indicates that latent mesangial IgA deposition is a relatively common phenomenon. Furthermore, patients with IgA nephropathy vary significantly with respect to progression and prognosis; some have a relatively good prognosis while others lose renal function, sometimes very rapidly. Thus, a variety of mechanisms may lay down deposits of IgA in glomerular mesangial areas, causing subsequent glomerular injuries and clinically active renal disease.

So far, we have identified five candidate genes that may be related to susceptibility to IgA nephropathy. On the basis of that information, we propose the potential mechanisms of $\operatorname{IgA}$ nephropathy indicated in Fig. 4, although it might be too preliminary, and more extensive biological and clinicopathological analysis would be required to substantiate this hypothesis. The onset of IgA nephropathy could be associated with antigens such as viruses, fungus, bacteria, or foods that are processed
Antigens (Virus, Fungus, Bacteria, Food etc.)

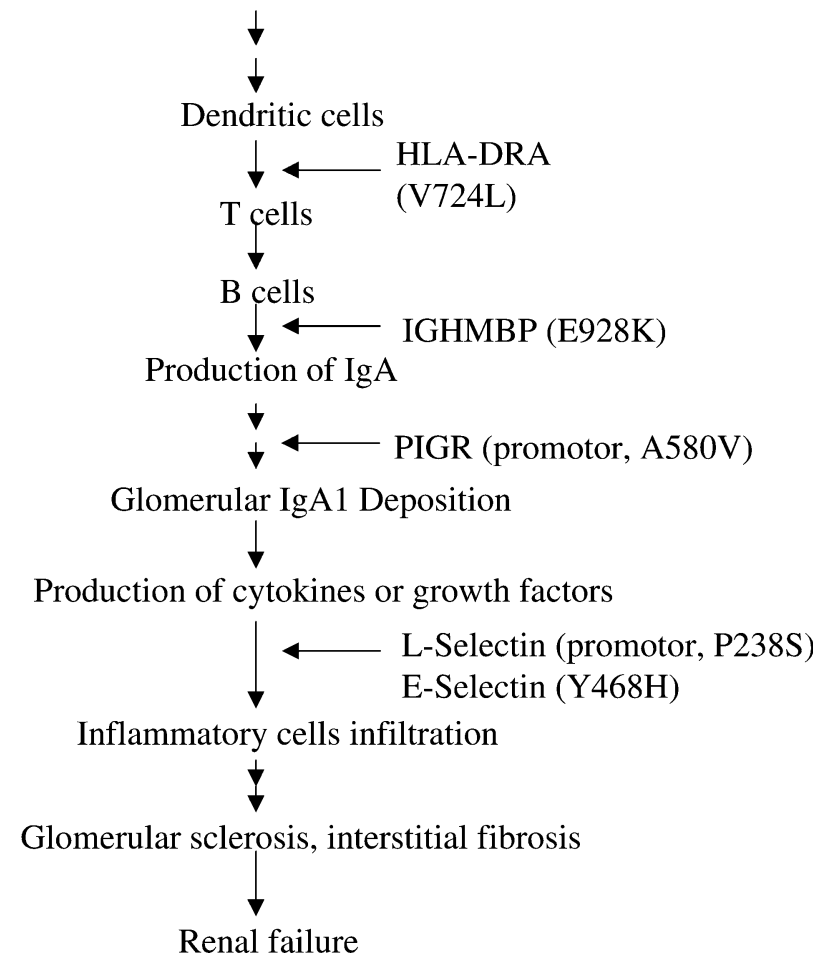

Fig. 4 Potential mechanism of IgA nephropathy based on results of case-control association studies using SNP markers for four candidate genes

and presented to $\mathrm{T}$ cells. HLA-DR, which regulates immune responses against protein antigens, is of great importance in the selection and activation of CD4-positive T cells; we identified the gene encoding HLA-DR earlier as a candidate susceptibility gene (Akiyama et al. 2002). HLA-DR molecules with the V724L substitution might account for individual differences in immune responses of $\mathrm{T}$ cells, which activate antibody-producing $\mathrm{B}$ cells. For its part, as noted above, the E928K variant of IGHMBP2 might influence a class-switch leading to increased serum IgA levels.

The third of our candidates, PIGR, is an integral membrane secretory component localized on the basolateral surface of secretory epithelial cells where it is thought to mediate the transepithelial transport of polymeric IgA. We showed earlier that a genetic variation in the promoter region of the PIGR gene caused an A580V substitution associated with IgA nephropathy and suggested that the $\mathrm{V}$ allele might affect binding of polymeric IgA to PIGR and cause deposition of mesangial IgA (Obara et al. 2003). IgA deposits in the kidney can trigger production of a variety of cytokines and growth factors by renal cells and by circulating inflammatory cells, leading to the characteristic histopathological features of mesangial-cell proliferation and depositions of immunoglobulin and complement in mesangial regions.

SELL and SELE genes encode cell-cell adhesion molecules involved in the leukocyte-endothelial cell 
interaction required for extravasation at sites of tissue injury. SELE is expressed predominantly in cytokineactivated endothelium, and SELL is present in circulating leukocytes. We reported that $\mathrm{Y} 468 \mathrm{H}$ in the SELE gene, as well as P238S-SELL and an SNP in the promoter region of $S E L L$, were strongly associated with IgA and suggested that these substitutions could affect the quality and/or quantity of gene products and possibly play a significant role in inflammatory changes leading to renal fibrosis and ultimately renal failure (Takei et al. 2002).

Although functional studies must be undertaken to determine how these genetic variations, now including E928K-IGHMBP2, can affect the onset and development of $\operatorname{IgA}$ nephropathy, the results of our genetic studies have suggested several potential mechanisms for investigation.

Acknowledgements We gratefully acknowledge assistance from Susumu Saito, Akihiro Sekine, and technicians at the SNP Research Center, The Institute of Physical and Chemical Research (RIKEN). This work was supported in part by Research for the Future Program Grant 00L01402 from the Japan Society for the Promotion of Science.

\section{References}

Akiyama F, Tanaka T, Yamada R, Ohnishi Y, Tsunoda T, Maeda S, Takei T, Obara W, Ito K, Honda K, Uchida K, Tsuchiya K, Nitta K, Yumura W, Nihei H, Ujiie T, Nagane Y, Miyano S, Suzuki Y, Fujioka T, Narita I, Gejyo F, Nakamura Y (2002) Single-nucleotide polymorphisms in the class II region of the major histocompatibility complex in Japanese patients with immunoglobulin A nephropathy. J Hum Genet 47:532-538

Berger J, Hinglasis N (1968) Les depots intercapillaries d'IgA-IgG. J Urol Nephrol 74:694-695

Conley ME, Cooper MD, Micheal AF (1980) Selective deposition of immunoglobulin A1 in immunoglobulin A nephropathy, anaphylactoid purpura nephritis, and systemic lupus erythematosus. J Clin Invest 66:1432-1436

Devlin B, Risch N (1995) A comparison of linkage disequilibrium measures for fine-scale mapping. Genomics 29:311-322

Donadio JV, Grande JP (2002) IgA nephropathy. N Engl J Med 347:738-748

Fukita Y, Mizuta TR, Shirozu M, Ozawa K, Shimizu A, Honjo T (1993) The human $S \mu b p-2$, a DNA-binding protein specific to the single-stranded guanine-rich sequence related to the immunoglobulin $\mu$ chain switch region. J Biol Chem 268:1746317470

Gharavi AG, Yan Y, Scolari F, Schena FP, Frasca GM, Ghiggeri GM, Cooper K, Amoroso A, Viola BF, Battini G, Caridi G, Canova C, Farhi A, Subramanian V, Nelson-Williams C, Woodford S, Julian BA, Wyatt RJ, Lifton RP (2000) IgA nephropathy, the most common cause of glomerulonephritis, is linked to 6q22-23. Nat Genet 26:354-357

Haga H, Yamada R, Ohnishi Y, Nakamura Y, Tanaka T (2002) Gene-based SNP discovery as part of the Japanese Millennium Genome Project: identification of 190,562 genetic variations in the human genome. Single-nucleotide polymorphism. J Hum Genet 47:605-610

Hsu SI, Ramirez SB, Winn MP, Bonventre JV, Owen WF (2000) Evidence for genetic factors in the development and progression of IgA nephropathy. Kidney Int 57:1818-1835

Koyama A, Igarashi M, Kobayashi M (1997) Natural history and risk factors for immunoglobulin A nephropathy in Japan. Research Group on Progressive Renal Diseases. Am J Kidney Dis 29:526-532

Kruglyak L (1999) Prospects for whole-genome linkage disequilibrium mapping of common disease genes. Nat Genet 22:139144

Lander E, Kruglyak L (1995) Genetic dissection of complex traits: guidelines for interpreting and reporting linkage results. Nat Genet 11:241-247

Liepinsh E, Leonchiks A, Sharipo A, Guignard L, Otting G (2003) Solution structure of the R3H domain from human Smubp-2. J Mol Biol 326:217-223

Mein CA, Barratt BJ, Dunn MG, Siegmund T, Smith AN, Esposito L, Nutland S, Stevens HE, Wilson AJ, Phillips MS, Jarvis N, Law S, de Arruda M, Todd JA (2000) Evaluation of single nucleotide polymorphism typing with invader on PCR amplicons and its automation. Genome Res 10:330-343

Miao M, Chan SL, Fletcher GL, Hew CL (2000) The rat ortholog of the presumptive flounder antifreeze enhancer-binding protein is a helicase domain-containing protein. Eur $\mathbf{J}$ Biochem 267:7237-7246

Molnar GM, Crozat A, Kraeft SK, Dou QP, Chen LB, Pardee AB (1997) Association of the mammalian helicase MAH with the pre-mRNA splicing complex. Proc Natl Acad Sci USA 94:7831-7836

Nielsen DM, Ehm MG, Weir BS (1998) Detecting marker-disease association by testing for Hardy-Weinberg disequilibrium at a marker locus. Am J Hum Genet 63:1531-1540

Obara W, Iida A, Suzuki Y, Tanaka T, Akiyama F, Maeda S, Ohnishi Y, Yamada R, Tsunoda T, Takei T, Ito K, Honda K, Uchida K, Tsuchiya K, Yumura W, Ujiie T, Nagane Y, Nitta K, Miyano S, Narita I, Gejyo F, Nihei H, Fujioka T, Nakamura Y (2003) Association of single-nucleotide polymorphisms in the polymeric immunoglobulin receptor gene with immunoglobulin A nephropathy (IgAN) in Japanese patients. J Hum Genet 48:293-299

Ohnishi Y, Tanaka T, Ozaki K, Yamada R, Nakamura Y (2001) A high-throughput SNP typing system for genome-wide association studies. J Hum Genet 46:471-477

Scolari F (2003) Inherited forms of IgA nephropathy. J Nephrol $16: 317-320$

Suzuki K, Honda K, Tanabe K, Toma H, Nihei H, Yamaguchi Y (2003) Incidence of latent mesangial IgA deposition in renal allograft donors in Japan. Kidney Int 63:2286-2294

Takei T, Iida A, Nitta K, Tanaka T, Ohnishi Y, Yamada R, Maeda S, Tsunoda T, Takeoka S, Ito K, Honda K, Uchida K, Tsuchiya K, Suzuki Y, Fujioka T, Ujiie T, Nagane Y, Miyano S, Narita I, Gejyo F, Nihei H, Nakamura Y (2002) Association between single-nucleotide polymorphisms in selectin genes and immunoglobulin A nephropathy. Am J Hum Genet 70:781-786

Trascasa ML, Egido J, Sancho J, Hernando L (1980) IgA glomerulonephritis (Berger's disease): evidence of high serum levels of polymeric IgA. Clin Exp Immunol 42:247-254

Zhang Q, Wang YC, Montalvo EA (1999) S $\mu$ bp-2 represses the Epstein-Barr virus lytic switch promoter. Virology 255:160-170 